\title{
Dyslipidemia and atherosclerotic carotid artery stenosis
}

\author{
Yoichi Miura, Hidenori Suzuki \\ Department of Neurosurgery, Mie University Graduate School of Medicine, Tsu 514-8507, Japan.
}

Correspondence to: Dr. Hidenori Suzuki, Department of Neurosurgery, Mie University Graduate School of Medicine, 2-174 Edobashi, Tsu 514-8507, Japan. E-mail: suzuki02@clin.medic.mie-u.ac.jp

How to cite this article: Miura Y, Suzuki H. Dyslipidemia and atherosclerotic carotid artery stenosis. Vesse/ P/us 2019;3:1. http:// dx.doi.org/10.20517/2574-1209.2018.69

Received: 21 Oct 2018 First Decision: 18 Dec 2018 Revised: 31 Dec 2018 Accepted: 31 Dec 2018 Published: 21 Jan 2019

Science Editor: Alexander N. Orekhov Copy Editor: Cui Yu Production Editor: Huan-Liang Wu

\begin{abstract}
Carotid artery atherosclerosis or stenosis is frequently present at the carotid bifurcation or the internal carotid artery, accounting for at least $20 \%$ of all ischemic strokes. High levels of serum total cholesterol and low-density lipoprotein cholesterol are established risk factors for genesis and progression of atherosclerotic lesions through various mechanisms. In addition, accumulating evidence has shown that a high level of triglyceride is associated with increased atherosclerosis risks. The so-called "vulnerable plaque" with a large lipid core, thin fibrous cap and intra-plaque hemorrhage tends to cause subsequent thromboembolic ischemic events. Statins are known not only to lower serum cholesterol levels but also to promote plaque stabilization via pleiotropic effects such as reducing subclinical systemic inflammation, endothelial activation, leukocyte intra-plaque infiltration, and increasing intimal smooth muscle cell migration. This article discusses the mechanisms of atherosclerosis formation induced by dyslipidemia and the role of lipid-lowering agents including statins in patients with symptomatic and asymptomatic atherosclerotic carotid artery stenosis.
\end{abstract}

Keywords: Atherosclerosis, carotid artery stenosis, lipid-lowering agent

\section{INTRODUCTION}

Carotid artery (CA) stenosis is caused by local thickening of CA wall due to atherosclerosis, and has a predilection for the CA bifurcation or the internal CA. The prevalence of significant CA stenosis is reported to be $7 \%-9 \%$ in the general population ${ }^{[1]}$. The high prevalence was observed in association with acute ischemic stroke (60\%), coronary heart disease (18\%), and atherosclerosis (11\%) ${ }^{[2]}$. A thromboembolism from CA atherosclerotic plaque causes at least $20 \%$ of all ischemic strokes ${ }^{[3]}$. The progression of CA atherosclerosis was promoted by dyslipidemia, hypertension, smoking, diabetes, and certain hemodynamic features

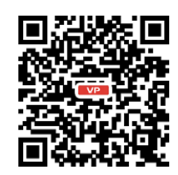


including turbulent blood flow or low wall shear stress ${ }^{[4]}$. Accumulating evidences indicate that symptomatic $\geq 70 \%$ CA stenosis should undergo expedited surgery, and most of the guidelines recommend carotid endarterectomy (CEA) within 2 weeks of minor ischemic strokes or transient ischemic attacks (TIAs). In contrast, some guidelines also recommend CEA for asymptomatic CA stenosis, but surgical or interventional therapies for asymptomatic CA stenosis are a weaker recommendation ${ }^{[5]}$. This is because recent advance in medical therapies has reduced the stroke risk in an asymptomatic CA stenosis. Indeed, a large prospective cohort study reported that asymptomatic $\geq 50 \%$ CA stenosis was associated with an annual rate of $<1 \%$ ischemic strokes ${ }^{[6]}$. According to meta-analyses, an annual rate of ischemic strokes in asymptomatic severe CA stenosis was decreased to $1.13 \%$ after 2000 with medical therapy alone, while that was $2.83 \%$ before $2000^{[7]}$. The remarkable thing is that the time of decreases in ischemic stroke risks in CA stenosis overlaps with that of increases in modern intensive medical treatment including statin medications and the improved control of hypertension.

Several lipid-lowering agents such as a statin are well known to be effective for the primary or secondary stroke prevention ${ }^{[8-10]}$. Statin exerts not only lipid-lowering effects but also pleiotropic effects including the reduction of inflammatory reactions, endothelial cell (EC) activation, and smooth muscle cell (SMC) proliferation $^{[11]}$. As for the association between statins and atherosclerotic CA stenosis, statins are well known to decrease CA intima-media thickness (IMT) ${ }^{[12-15]}$, suppress CA plaque progression ${ }^{[16,17]}$, and improve CA plaque vulnerability ${ }^{[18,19]}$.

This article focuses on mechanisms of CA atherosclerosis development, by which dyslipidemia induces, and discusses potential therapeutic roles of stains as well as non-statin lipid-lowering agents and non-drug therapies for atherosclerotic CA stenosis.

\section{THE FORMATION OF ATHEROSCLEROTIC CA PLAQUE [Figure 1]}

The mechanisms of atherosclerotic formation in CA are similar to those in other arteries. A hemodynamic shear stress triggers EC dysfunction and induces SMC accumulations in the subendothelial space, initiating atherosclerosis formation at arterial branch sites as intimal cell masses ${ }^{[2,21]}$. Physical or metabolic injuryinduced disturbance of EC integrity makes ECs transduce hemodynamic stress into biochemical signals, which change the expressions of cell adhesion molecules (CAMs) and other cell surface receptors to alter blood cell adhesion ${ }^{[20]}$. Several mediators including reactive oxygen species induce CAMs such as intercellular adhesion molecule (ICAM)-1, vascular CAM (VCAM)-1, and endothelial-leukocyte adhesion molecules on $\mathrm{ECs}^{[21]}$. The first step of atherogenesis is that low-density lipoprotein (LDL) cholesterol (Cho) (LDL-C) enters into the subendothelial spaces, and is trapped by a high affinity to the glycoprotein molecules at the lesion ${ }^{[22]}$. Although LDL particles cannot penetrate the junctions between ECs due to their too large molecular size, most of circulating LDLs can be transported across the EC by receptormediated or nonspecific uptake into micropinocytic channels. Each EC has receptors for both LDLs and the modified forms. A free receptor modifies and oxidizes LDL into modified forms of LDL such as oxidized LDLs (ox-LDLs), and ox-LDLs promote transendothelial migration of monocytes into the subendothelial spaces, which is guided by chemokines. Moreover, ox-LDLs induce the differentiation of monocytes into macrophages. Macrophages develop receptors for ox-LDLs to become lipid-laden foam cells through the receptor-mediated incorporation of ox-LDLs ${ }^{[21]}$. Thus, Cho is trapped within the arterial walls, which is the hallmark of early-stage atherosclerotic lesion formation. Macrophage also induces local inflammatory reactions within the vessel walls, which are promoted by cytokines, activated helper $\mathrm{T}$ cells, and activators of scavenger receptors ${ }^{[23]}$. Accumulated lipid-laden foam cells in the tunica intima in the artery wall mature fatty streaks and produce a lesion, which is covered with a fibrous cap consisting of macrophages, SMCs, and extracellular matrix (ECM) components including collagen, elastin and proteoglycans ${ }^{[24]}$. The fibrous cap is a layer of connective tissues, and separates a lipid-rich core from arterial lumen, forming atherosclerotic plaques. When chronic inflammation is present, foam cells are persistently recruited, and apoptosis and 


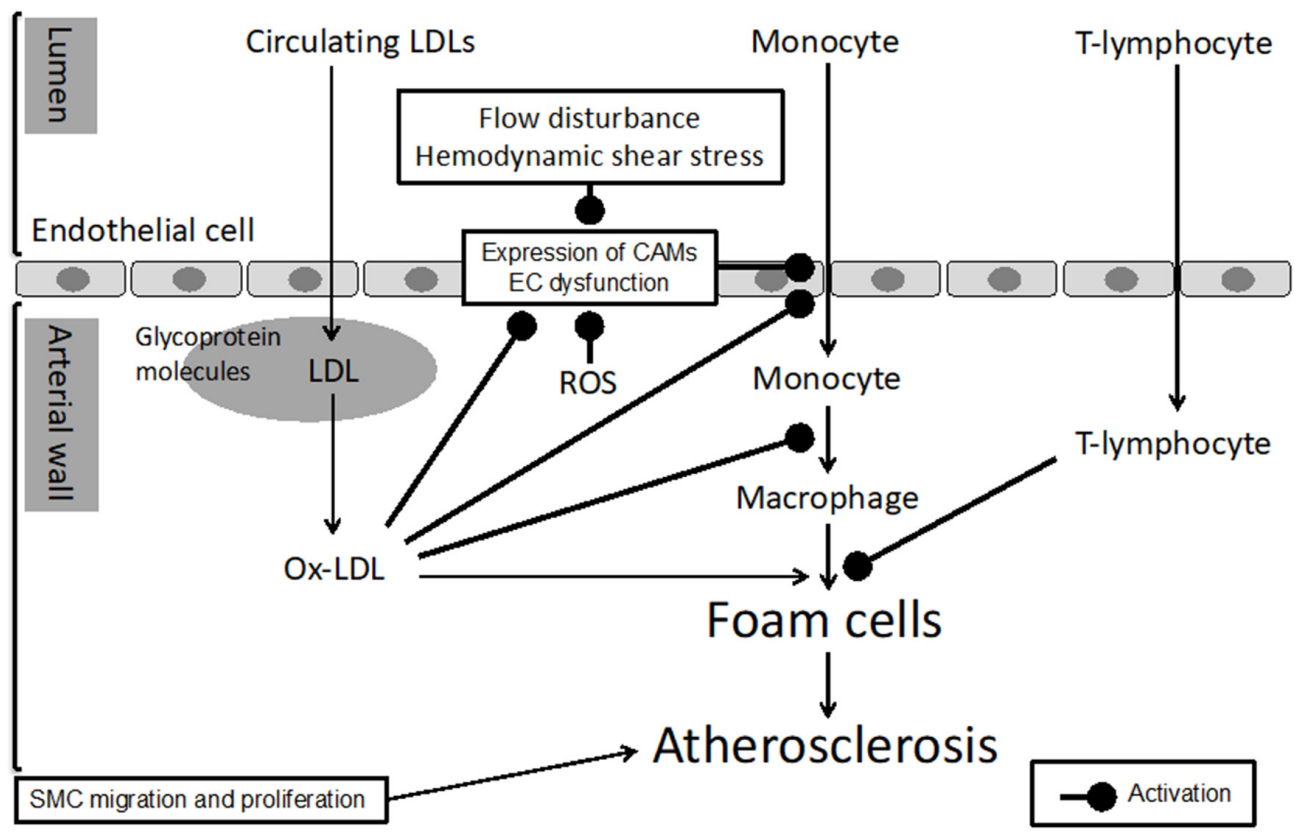

Figure 1. Formation of atherosclerosis. Atherosclerosis begins at the sites of EC damage or dysfunction, and develops with inflammatory reactions and dynamic interactions among plasma molecules including LDL and Ox-LDL, monocytes and macrophages. EC: endothelial cell; LDL: low density lipoprotein; Ox-LDL: oxidized LDL; CAM: cell adhesion molecule; ROS: reactive oxygen species; SMC: smooth muscle cell

ECM degradation by matrix metalloproteinases (MMPs) are promoted and associated with more necrotic environment within atherosclerotic plaques. As a result, atherosclerotic lesions are furthermore developed and matured.

\section{CA PLAQUE VULNERABILITY}

An atherosclerotic CA plaque consists of a lipid core with inflammatory cell infiltrations and a fibrous cap, and is classified into stable and unstable or vulnerable ones. A stable plaque is characterized by a thicker fibrous cap, which prevents plaques from rupture. In contrast, the characteristics of unstable or vulnerable plaque is intra-plaque hemorrhage and a large lipid core covered with a thin fibrous cap, which contains less ECM and SMCs, and is often associated with the infiltration of inflammatory cells and the secretion of MMPs and cytokines ${ }^{[25]}$. Several reports have shown that a rich network of small vessels, that is, vasa vasorum, is interweaved into the ECM of most of mature plaques ${ }^{[26]}$. Unstable or vulnerable plaque is more likely to rupture, causing thromboembolic strokes. Intra-plaque hemorrhage is also known as a predictor for thromboembolic strokes and the recurrence. As well, strong correlations are observed between intra-plaque hemorrhage and plaque rupture, and symptomatic CA stenosis was more frequently associated with intraplaque hemorrhage compared with asymptomatic CA stenosis $(74 \% \text { vs. } 32 \%)^{[27]}$.

Many atherosclerotic mediators play a role in CA plaque vulnerability ${ }^{[28-30]}$. Morgan et al.$^{[28]}$ reported the relationships between MMPs-1 or -12 and CA plaque instability: MMP-1 was upregulated more in a CA plaque with a thin fibrous cap compared with that with a thick fibrous cap, and MMP-12 was induced more in a ruptured CA plaque than a CA plaque with no disruption of a fibrous cap. Montecucco et al. ${ }^{[29]}$ demonstrated that the down-regulation of cannabinoid receptor type 2 that prevents neutrophil release of MMP-9 caused an increase in vulnerability in a symptomatic CA plaque. It was also found that antiapolipoprotein (Apo) A-1 auto-antibodies played a role in an increase in histological features of plaque vulnerability in severe CA stenosis ${ }^{[29]}$. More recently, Rao et al. ${ }^{[30]}$ revealed that triggering receptor expressed on myeloid cells (TREM)-1 related to MMPs-1 and -9 was increased in a symptomatic CA plaque, suggesting a potential role of TREM-1 in CA plaque destabilization. 


\section{DYSLIPIDEMIA AND ATHEROSCLEROSIS}

\section{Total Cho}

Numerous epidemiology and clinical studies have demonstrated that dyslipidemia is a major risk factor for atherosclerosis formation ${ }^{[2,21]}$. Hypercholesterolemia increases levels of cellular free Cho about 2- to 4-fold in vascular $\mathrm{ECs}^{[31]}$. As a result, a high level of free Cho in ECs induces changes in the plasma membrane Cho content and the composition of lipid rafts, leading to altering membrane function and therefore affecting cell function in $\mathrm{SMCs}^{[32]}$. High levels of free Cho also increase reactive oxygen species generation via various mechanisms ${ }^{[31,33]}$. Increased reactive oxygen species generation causes EC dysfunction in terms of reducing nitric oxide bioavailability and uncoupling endothelial nitric oxide synthase (eNOS). In addition, hypercholesterolemia activates nicotinamide adenine dinucleotide phosphate (NADPH) oxidases, xanthine oxidases and myeloperoxidases, resulting in reactive oxygen species generation ${ }^{[21]}$. Hypercholesterolemia also promotes the secretion of a proinflammatory cytokine such as tumor necrosis factor- $\alpha$, interleukin$1 \beta$, and interferon $-\gamma$, as well as mitochondrial and NADPH oxidase-generated reactive oxygen species ${ }^{[21]}$. In the presence of hypercholesterolemia, caveolin, an essential protein component of caveolae, binds to eNOS, leading to an inactivity of $\mathrm{eNOS}^{[21]}$.

\section{LDL}

LDL interactions with the endothelium have been closely associated with accelerated atherosclerosis ${ }^{[20,21]}$. LDLs and ox-LDLs activate EC NADPH oxidases, generating reactive oxygen species ${ }^{[2,33]}$. LDLs and ox-LDLs also cause increases in the binding of eNOS to CD36, and consequently the attenuation of eNOS activity and the displacement of the protein from EC caveolae are induced ${ }^{[34]}$. Additionally, the interactions between eNOSs and NADPH oxidases determine the production of nitric oxide and reactive oxygen species, because nitric oxide produced adjacent to NADPH oxidases is scavenged by reactive oxygen species ${ }^{[35]}$. Furthermore, native LDLs increase the expressions of ICAM-1, VCAM-1, and p-selectin ${ }^{[21]}$.

\section{Triglyceride and TG-rich lipoprotein}

Triglyceride (TG) is a major component of TG-rich lipoprotein (TGRL) including chylomicrons (CMs), very LDLs (VLDLs) and their remnants. TG and TGRL contribute directly to the development and progression of atherosclerotic plaque. While CMs and larger VLDLs cannot penetrate the arterial walls, their smaller TGRL remnants not only penetrate the arterial intima, but also promote the binding and retention to connective tissue matrix ${ }^{[36]}$. This transcytosis involvement in the transport system is restricted to a lipoprotein with a diameter of $\leq 60-70 \mathrm{~nm}$. TGRL remnants carry about 40 times more Cho per particle compared with LDLs, due to their larger size ${ }^{[36]}$. Accumulation of CMs and VLDLs with abundant Apo E have been shown in an atherosclerotic plaque. Macrophages directly take up such particles, and resultantly have massive Cho loading, leading to the formation of foam cells.

TG and TGRL also accelerate the atherogenesis through an indirect mechanism, particularly that involving binding and lipolysis at the arterial walls ${ }^{[37-40]}$. TGRL increases the production of reactive oxygen species, secretion of tumor necrosis factor- $\alpha$, and expressions of CAMs ${ }^{[37]}$. A high level of TGs leads to TGRLs enriched with Apo C-III, and influences signaling pathways, which lead to activation of nuclear factor $(\mathrm{NF})-\kappa \mathrm{B}$ and upregulation of inflammatory mediators, causing the development of fatty streaks and the advancement of atherosclerosis ${ }^{[38]}$. CM remnants migrate to the subendothelial space, activate leukocytes, and accelerate the formation of foam cells: CM also activates monocytes, and enhances migration of monocytes and postprandial neutrophils ${ }^{[39]}$. TG in the presence of an elevated concentration of VLDL generates small dense LDL, which is particularly atherogenic, since these particles are retained preferentially by the arterial walls. Furthermore, elevated TG is linked with procoagulant states by increasing factor VII, and activating factor VII phospholipid complexes, factor X, factor XII, tissue plasminogen activator inhibitor, and thrombin generation ${ }^{[21,40]}$. 
The proatherogenic mechanisms associated with TG and TGRL are rather complicated and need to be further explored. However, current knowledge and accumulating evidences of clinical studies indicate that therapies lowering TG levels can be one of important treatment strategies to suppress atherosclerotic formation and ischemic stroke risks.

\section{High-density lipoprotein Cho}

High-density lipoprotein Cho (HDL-C) is potentially highly protective for atherosclerosis. HDL-C is classically known to reverse Cho transport: that is, HDL-C removes excess Cho from foam cells within peripheral tissues, and delivers it to liver for metabolism ${ }^{[41]}$. In addition, recent reports have shown that HDL-C exerts a lot of other functions, which reduce atherosclerotic development ${ }^{[41-43]}$. Beneficial effects of HDL-C against atherogenesis may come from its protective effects against the generation of reactive oxygen species and VCAMs ${ }^{[42]}$. Reconstituted HDL-C suppresses the activation of leukocyte NADPH oxidases $^{[43]}$. The effects of HDL-C may contribute to protecting vascular injuries, resulting in the suppression of atherosclerosis. HDL-C inhibits the expressions of cytokine-induced CAMs, and then suppresses the adhesion of monocytes to ECs ${ }^{[44]}$. Paraoxonase-containing HDL-C suppresses oxidation of LDL-C, and prevents expressions of monocyte chemotactic protein-1 ${ }^{[45]}$. As well, LDL-C was reported to increase monocyte chemotactic protein-1 expressions and cause 7-fold increases in monocyte migration into the subendothelial space, whereas purified HDLs inhibited the monocyte migration by $91 \%{ }^{[46]}$.

HDL-C protects erythrocytes against the generation of procoagulant activity, and enhances anticoagulant activities of protein $S^{[47]}$. HDL-C is also positively correlated with plasminogen activator inhibitor-1 activity $^{[21]}$. Furthermore, HDL-C inhibits thrombin-induced binding of fibrinogen to platelets and suppresses the platelet aggregation ${ }^{[48]}$. Thus, HDL-C can reduce blood clot formation, and therefore high levels of HDL-C can prevent ischemic stroke.

\section{DYSLIPIDEMIA AND STROKE}

A high level of serum total Cho (TC) and LDL-C is well known to be major risk factors for ischemic stroke. LDL-C-lowering therapies have attracted extensive attention, and have been a cornerstone of the primary and secondary prevention for ischemic stroke ${ }^{[49]}$. Recently, higher TG levels also have been repeatedly reported to increase atherosclerotic risks ${ }^{[50]}$. A meta-analysis study was conducted to investigate the association between lipid levels induced by lipid-modifying drugs and stroke risks, and revealed that higher levels of TG at baseline had a higher incidence of ischemic stroke occurrence (adjusted relative risk, 1.05 per $10 \mathrm{mg} / \mathrm{dL}$ increase) in placebo groups in 64 randomized clinical trials ${ }^{[50]}$. In contrast, HDL-C levels are inversely associated with risks for the development of atherosclerosis and ischemic stroke ${ }^{[41,51,52]}$. According to a systematic review of 18 prospective studies investigating relationships between HDL-C levels and stroke risks, each $10 \mathrm{mg} / \mathrm{dL}$ increase in HDL-C levels decreased risks for ischemic strokes ranging from $11 \%-15 \%{ }^{[52]}$. Some reports suggested that the negative association between HDL-C levels and ischemic stroke risks is more dependent on HDL-C sub-fractions rather than total levels of HDL-C. HDL sub-fractions have been classified on their size and relative concentrations of Cho, and each sub-fraction is known to have different biological activities, biochemical properties, and vascular metabolisms ${ }^{[41,53]}$. HDL-2 and HDL-3 are major circulating sub-fractions in the peripheral blood ${ }^{[53]}$. In contrast with HDL-2, smaller dense protein-rich HDL-3 appears to suppress oxidation of LDLs and thereby to prevent atherosclerosis ${ }^{[53]}$. It was demonstrated that HDL sub-fractions differently influenced CA diseases in the Northern Manhattan study: HDL-2 and plaque thickness were positively related, while HDL-3 and plaque area were inversely associated ${ }^{[54]}$. In another prospective study, however, small-size HDL-C and medium-size HDL-C were both associated with lower incidences of stroke occurrence, in particular as to lacunar infarcts and hemorrhagic strokes ${ }^{[55]}$. On the other hand, large-size HDL-C did not influence the stroke risks ${ }^{[55]}$. More studies are thus needed to clarify and understand the association between HDL sub-fractions and the risk of strokes. Another important finding is that lower levels of TC and LDL-C were associated with higher risks of intracranial 
hemorrhage ${ }^{[56,57]}$. In a previous meta-analysis study, an inverse dose-response association was shown between TC levels and hemorrhagic stroke risks [odds ratio (OR), 0.85 per $1 \mathrm{mmol} / \mathrm{L}$ increase ${ }^{[56]}$. A more recent systematic review also showed that hemorrhagic stroke was increased under low TC conditions: East Asian ethnic status favors the development of subarachnoid hemorrhage, and non-East Asian ethnic status is predisposed to intracerebral hemorrhage ${ }^{[57]}$.

As to the relationships between dyslipidemia and the subtypes of ischemic strokes, dyslipidemia has a stronger association with ischemic stroke due to atherosclerotic large artery diseases: it was reported that the association between Cho levels and risks of atherosclerotic stroke was the highest $(\mathrm{OR}, 3.2)^{[51]}$, and the findings were confirmed in other studies ${ }^{[49]}$. On the other hand, there are no or obscure associations between dyslipidemia and other ischemic stroke subtypes: an association between dyslipidemia and lacunar strokes was shown in some studies but not all studies ${ }^{[51]}$, and most studies failed to show any association of dyslipidemia with cardioembolic infarction ${ }^{[49,51]}$.

\section{DYSLIPIDEMIA AND CA STENOSIS}

The risk factors for CA stenosis are similar to those for other vascular diseases, and the relationship between dyslipidemia and CA stenosis is well known. Mathiesen et al ${ }^{[58]}$ reported that TC, HDL-C (inversely), fibrinogen, systolic blood pressure, and current smoking were independent factors for the development of CA stenosis. The determinants of CA IMT were LDL-C levels, systolic blood pressure, body mass index and smoking in childhood, and systolic blood pressure, body mass index and smoking in adulthood ${ }^{[59]}$. Other previous studies reported that the progression of CA atherosclerosis was associated with a higher level of TC, LDL-C, or a lower level of HDL-C ${ }^{[60]}$. In patients with type 2 diabetes, regression of CA IMT was observed when LDL-C and systolic blood pressure were reduced to a lower target ${ }^{[61]}$.

Several studies investigated if TG can be a risk factor for the progression of CA stenosis ${ }^{[60,62]}$. In patients with diabetes, the CA atherosclerosis progression tended to occur more frequently when fasting TG levels were higher ${ }^{[60]}$. Recently, Kitagami et al. ${ }^{[62]}$ reported that a higher level of TG was an independent risk factor for the progression of CA atherosclerosis in patients with moderate to severe CA stenosis who were treated with CA stenting (CAS), CEA, or other treatments under well-controlled LDL-C levels. The findings suggest that to control TG levels at least within the normal limits is an important management strategy for CA stenosis ${ }^{[62]}$. However, to our knowledge, no studies have revealed the relationships between the degree of changes in TG levels and the rate of CA IMT progression. It is expected that some studies investigate if therapeutic reduction of TG levels can suppress the risks of progression of CA atherosclerosis or stenosis.

\section{LIPID-LOWERING AGENTS AND CA STENOSIS [Figure 2]}

\section{Statin}

Statin inhibits the synthesis of Cho in liver and thereby increases the uptake of Cho by liver, resulting in a decrease in circulating lipid levels: thus, statin has been extensively reported to exert a lipid-lowering effect.

Experimental studies have demonstrated protective effects of statins on ischemic stroke ${ }^{[63-65]}$. For example, rosuvastatin upregulated eNOS and protected ischemic brain in middle cerebral artery occlusion (MCAO) mice ${ }^{[63]}$; atorvastatin reduced brain edema via the suppression of aquaporin 4 expression in MCAO rats ${ }^{[64]}$; and simvastatin protected cerebrum from ischemic and reperfusion injury by decreasing the expressions of $\mathrm{Ca}^{2+} /$ calmodulin-dependent protein kinase II and aquaporin 4 in MCAO rats ${ }^{[65]}$.

In clinical settings, there are good evidences that statin reduces risks of ischemic strokes by about 30\%, and that statins are effective for the primary prevention of ischemic strokes in the elderly and patients with diabetics, or hypertension ${ }^{[8]}$. In addition, statins are reported to have beneficial effects on the secondary prevention for TIAs and ischemic strokes: statin administration significantly reduced the risk of fatal or 


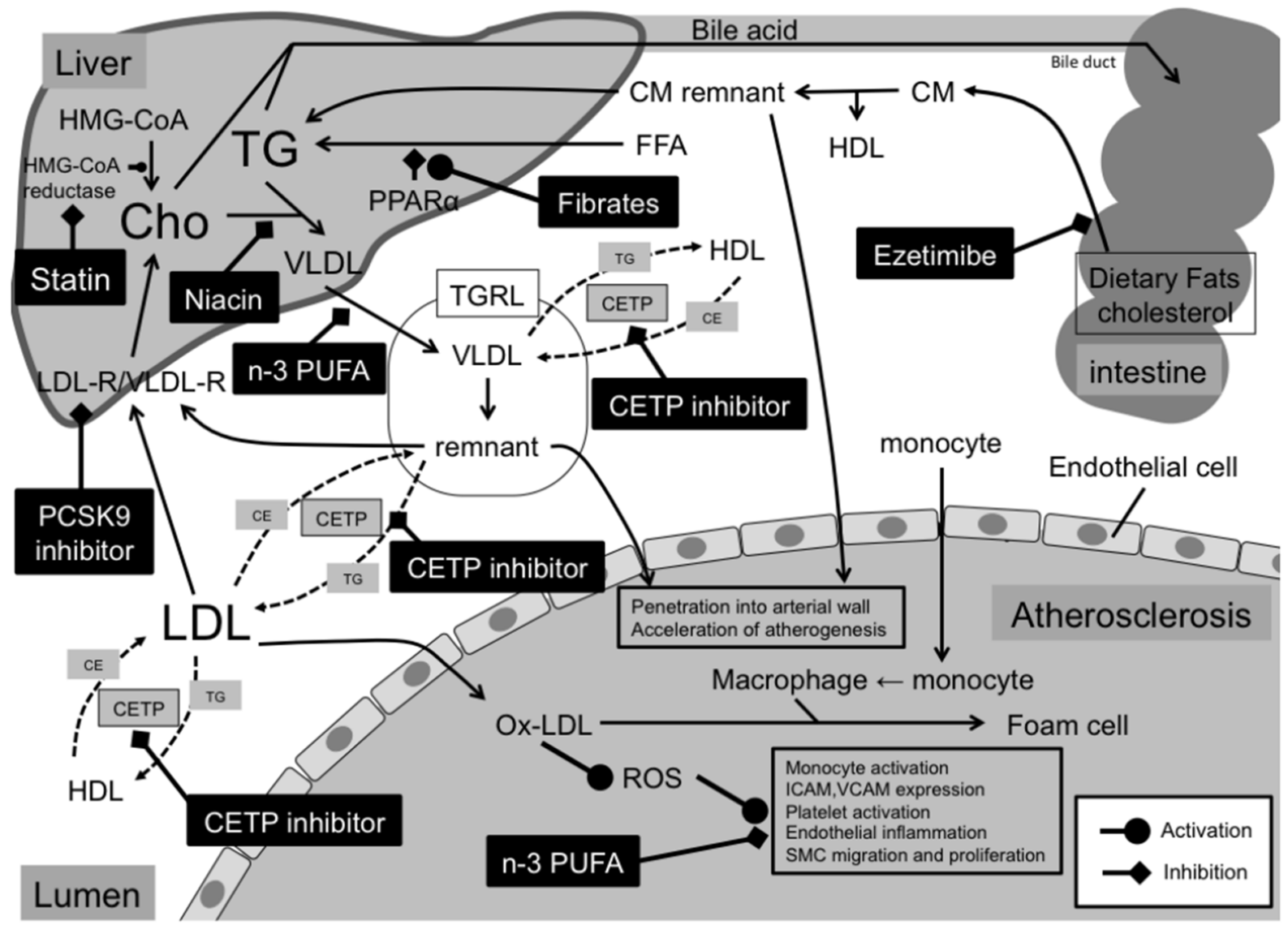

Figure 2. Metabolic pathways for cholesterol (Cho) and the main acting points of lipid-lowering agents. Statin is a 3-hydroxy-3methylglutaryl coenzyme A (HMG-CoA) inhibitor, and exerts lipid-lowering effects by inhibiting the synthesis of Cho in liver and pleiotropic effects such as anti-inflammation and improvement of endothelial dysfunction. Niacins modify plasma lipid levels by inhibition of lipolysis in adipose tissues. Fibrates reduce triglyceride (TG) levels and increase high-density lipoprotein (HDL) Cho (HDL-C) levels via various mechanisms including the inactivation of peroxisome proliferator activated receptor (PPAR) $\alpha$, which increases the oxidation of free fatty acid (FFA) in liver and reduces the hepatic synthesis of TG and expression of lipoprotein lipase. Omega-3 polyunsaturated fatty acids (PUFAs), the major component of fish oil, are widely used as a TG-lowering therapy and have been found against oxidative stress and inflammation. Ezetimibes reduce low-density lipoprotein (LDL) Cho (LDL-C) levels by inhibition of the absorption of Cho from intestines. Cho ester (CE) transfer protein (CETP) inhibitors increase HDL-C levels by the inhibition of CETP, which promotes the net effects on the equilibration of both CE and TG among all lipoprotein particles. Proprotein convertase subtilisin-kexin type 9 (PCSK9) inhibitors remove LDL-C from plasma by the inhibition of PCSK9, which is a hepatic protease that decreases hepatic LDL receptor (LDL-R) leading to an increase in the plasma concentration of LDL-C. CM: chylomicron; ICAM: intercellular adhesion molecule; Ox-LDL: oxidized LDL; ROS: reactive oxygen species; SMC: smooth muscle cell; TGRL: TG-rich lipoprotein; VCAM: vascular cell adhesion molecule; VLDL: very LDL; VLDL-R: VLDL receptor

nonfatal strokes by $16 \%{ }^{[9]}$ and prevented the development of atherothrombotic infarction even after adjusting LDL-C levels at baseline [adjusted hazard ratio (HR), 0.39] ${ }^{[10]}$. The American Heart Association guideline states that statin treatment is reasonable for reducing LDL-C levels to less than or equal to $70 \mathrm{mg} / \mathrm{dL}$ in a patient with an atherosclerotic disease at the extracranial CA or vertebral artery who has sustained ischemic strokes ${ }^{[66]}$.

Statins are well known not only to decrease IMT and to suppress plaque progression in the CA, but also to improve plaque vulnerability in the CA as described below. These effects might be exerted by their pleiotropic effects, which include the inhibition of subclinical systemic inflammation, EC activation, and intra-plaque infiltrations of leukocytes, as well as an increase in protective SMC migration into plaques ${ }^{[11]}$.

\section{Statin and CA IMT}

Stains were for the first time reported to play a role in reducing CA IMT in coronary heart disease patients with hypercholesterolemia ${ }^{[12]}$. After that, several randomized clinical trials revealed that statins have 
beneficial and preventive effects against the progression of CA IMT ${ }^{[13-15]}$. According to meta-analyses of 7 clinical trials of statins, statin treatments contributed to a mean of $-0.012 \mathrm{~mm} /$ year reduction in CA IMT ${ }^{[13]}$. In the subsequent meta-analyses of 11 clinical trials of statins, the mean differences of changes in carotid IMT between statin and placebo therapies were $-0.040 \mathrm{~mm} / 25.6$ months $(P<0.001)^{[14]}$. As regards dosages, intensive lipid-lowering treatments may result in a more regression of CA IMT compared with moderate lipid-lowering treatments in patients with familial hypercholesterolemia ${ }^{[67]}$.

\section{Statin and CA plaque volume}

Ainsworth et al. ${ }^{[17]}$ used 3-dimensional ultrasound scans and evaluated CA plaque volume after 3 months treatment with randomly assigned placebo or $80 \mathrm{mg} /$ day of atorvastatin in 38 patients with asymptomatic > 60\% CA stenosis: atorvastatin treatments decreased the plaque volume, although placebo treatments caused a tendency for the plaque volume to increase. Corti et al. ${ }^{[16]}$ used magnetic resonance imaging (MRI), and found that 2 years simvastatin treatments induced a significant regression in an asymptomatic plaque in both aorta and $\mathrm{CA}$ in hypercholesterolemic patients. In regard to dosages, the same group investigated effects of doses of 20 or $80 \mathrm{mg} /$ day simvastatin treatments for a mean follow-up of 18.1 months on CA plaques in 51 patients, and showed no differences in vessel wall changes between the two dosages ${ }^{[68]}$. In another clinical trial, 43 patients with asymptomatic 16\%-79\% CA stenosis were treated with randomly assigned low (5 mg/day) or high ( 40 or $80 \mathrm{mg} /$ day) dosages of rosuvastatin for 2 years, and showed no changes in CA plaque volume between the 2 treatment groups on duplex ultrasound ${ }^{[69]}$. In the trial, however, all plaques with a lipid-rich necrotic core at baseline were decreased by a mean of $41 \%$ with the statin treatments ${ }^{[69]}$. It was also demonstrated in patients with 24 statin-naïve newly diagnosed stable coronary artery diseases that 3 months open-label statin treatments induced a $3.1 \%$ reduction in the carotid plaque index (normalized vessel wall area) ${ }^{[70]}$.

\section{Statin and CA plaque vulnerability}

The relationships between statin treatments and CA plaque vulnerability were investigated previously ${ }^{[18,19]}$. Tang et al. ${ }^{[18]}$ estimated the extent of inflammations in CA plaques using ultra-small supermagnetic iron oxide enhanced carotid MRI, which visualizes the infiltrations of macrophages in human CA atheroma in vivo, and reported that 3 months treatments with a high dose ( $80 \mathrm{mg} / \mathrm{day})$, but not a low dose $(10 \mathrm{mg} / \mathrm{day})$ of atorvastatin decreased intra-plaque inflammations. Mujaj et al. ${ }^{[19]}$ reported that high-dosage statins have beneficial influences on the compositions of CA atherosclerosis: that is, statins shifted vulnerable plaques with a lipid core to more stable calcified plaques. Lenglet et al ${ }^{[7]]}$ revealed that statin treatment reduced serum levels of neutrophilic products, receptor activator of NF- $\mathrm{KB}$ ligand/osteoprotegerin ratio, osteopontin, and MMP-9 /pro-MMP-9 activity in severe CA stenosis patients having no history of ischemic stroke. Moreover, CA plaques on statin therapy exhibited an increase in collagen, and reduced levels of neutrophil infiltration and MMP-9 compared with untreated patients with asymptomatic severe CA stenosis ${ }^{[71]}$.

\section{Statin in CEA and CAS}

Statin therapy is reported to reduce perioperative risks of ischemic stroke, and to improve clinical outcomes in patients with CA stenosis undergoing CEA and CAS ${ }^{[72]}$. McGirt et al. ${ }^{[72]}$ investigated effects of statin treatment on the incidences of perioperative strokes and mortality in 1,566 patients with CA stenosis undergoing CEA, and showed that statins had a benefit to reduce perioperative strokes, TIAs and allcause mortality related to CEA. In the report, the usage of statins independently reduced the odds of stroke threefold (OR, 0.35) and death fivefold (OR, 0.20) ${ }^{[72]}$.

Periprocedural usage of statins may be more beneficial in patients with CA stenosis undergoing CAS, as high-pressure balloons and stents are applied during CAS and therefore thromboses and inflammations are likely to be developed within the vessel walls after CAS. Tanemura et al ${ }^{[73]}$ investigated the association between cerebral embolisms related to CAS and CA plaque characteristics using 3-dimensional T1-weighted gradient echo MRI, and revealed that vulnerable and large CA plaques had high risks for cerebral embolisms 
during CAS. In a recent meta-analysis of 8 studies that investigated effects of pre-CAS treatments with statins on periprocedural adverse events, statin treatments statistically reduced periprocedural risks of ischemic strokes $(\mathrm{OR}, 0.39)$ and death $(\mathrm{OR}, 0.30)$ in $\mathrm{CA}$ stenosis patients treated with $\mathrm{CAS}^{[74]}$.

\section{OTHER LIPID-LOWERING AGENTS [Figure 2]}

Classical non-statin lipid-lowering agents including niacin acids, fibrates and omega-3 fatty acids can improve lipid profiles. However, the benefit of these non-statin lipid-lowering agents has not been well established for the primary and secondary prevention of strokes. A meta-analysis including 78 clinical trials revealed that non-statin lipid-lowering agents such as fibrates, diet and other treatments did not reduce the risks of strokes (fibrates: OR, 0.98; diet: OR, 0.92; other treatments: OR, 0.81$)^{[75]}$.

Niacin can reduce peripheral blood levels of TG by approximately $35 \%$, decrease LDL-C levels by $10 \%-15 \%$, and increase HDL-C levels by up to $25 \%{ }^{[76]}$. Niacins may also enhance a beneficial effect such as vasoprotection and anti-inflammatory actions independent of its lipid-modifying activities ${ }^{[76]}$. Although Villines et al. ${ }^{[77]}$ revealed the regression of CA IMT thickening induced by niacin, its beneficial effects on the reduction of ischemic stroke risks remain uncertain. In a meta-analysis of 11 clinical studies including 9,959 patients, no significant association was found between niacin therapy and the risks of strokes $(\mathrm{OR}, 0.88)^{[78]}$.

Fibrate also lowers TG levels and increases HDL-C levels. Fibrate furthermore may exert pleiotropic effects via regulating the interaction with peroxisome proliferator activated receptor (PPAR) alpha, which influences vascular inflammation and thrombogenesis ${ }^{[79,80]}$. However, it is uncertain if fibrates can reduce risks of stroke event. In the clinical trial consisting of 2,531 men with coronary artery diseases and low levels of HDL-C, benzofibrate showed a $31 \%$ reduction in the risks of stroke ${ }^{[79]}$. On the other hand, a meta-analysis including 18 clinical trials with 45,058 patients showed no preventive effects of fibrates on stroke development ${ }^{[80]}$. Recently, pemafibrate has been drawn attention to as a novel selective PPAR alpha modulator. In the phase III clinical trial, pemafibrate significantly reduced TG levels from baseline by up to $45 \%$ with low incidence rates of adverse drug reactions ${ }^{[81]}$. In addition, the combination therapy of pemafibrate with pitavastatin exerted a robust reduction in a fasting level of TG by about $50 \%$ compared with the statin-monotherapy ${ }^{[81]}$. Another trial is ongoing to investigate the efficacy of pemafibrate on cereberovascular outcomes in patients with diabetes, and the results are awaited. It will be also necessary to verify whether pemafibrate has beneficial effects on the primary and secondary prevention of strokes in patients with CA stenosis.

Omega-3 polyunsaturated fatty acids (PUFAs), eicosapentaenoic acids (EPAs) and docosahexaenoic acids (DHAs) are approved as an adjunct to diet for lowering plasma TG via multiple metabolic pathways ${ }^{[82]}$. In a previous randomized clinical trial, 114 patients awaiting CEA were randomized to 3 groups taking placebo, fish oils (n-3 PUFA), and sunflower oils (n-6 PUFA): CA plaques in the n-3 PUFA treatment group had higher proportions of EPAs and DHAs, reduced infiltration of monocyte and macrophage, and a thicker fibrous cap compared with other groups ${ }^{[83]}$. In another randomized clinical trial including 121 patients awaiting CEA, the proportion of EPAs was higher in CA plaques treated with n-3 PUFAs compared to placebo $^{[84]}$. The remarkable thing is that the EPA content in plaque phospholipids had inverse association with plaque instability, plaque inflammation, and the number of $\mathrm{T}$ cells in the plaques ${ }^{[8]}$. CA atherosclerotic plaque in patients treated with n-3 PUFAs showed a lower level of messenger ribonucleic acids (mRNAs) for MMPs-7, $-9,-12$, interleukin- 6 and ICAM-1 ${ }^{[84]}$. Thus, the stability of plaques by increased n-3 PUFAs intake may induce the reductions in the risk of ischemic stroke.

Ezetimibe prevents the absorption of Cho from intestines, and therefore reduces a level of TC. In the clinical trial including 18,144 patients hospitalized for acute coronary syndrome, the combination therapy of 10 $\mathrm{mg} /$ day ezetimibe with $40 \mathrm{mg} /$ day simvastatin resulted in a significant reduction in stroke risks $(\mathrm{HR}, 0.936)^{[85]}$. 
Furthermore, in patients stabilized after acute coronary syndrome, the addition of ezetimibe to simvastatin reduced the incidence of ischemic strokes ( $\mathrm{HR}, 0.52)$ : the preventive effects on ischemic strokes were remarkable in patients with prior strokes ${ }^{[8]}$.

Cholesteryl ester transfer protein (CETP) inhibitors increase a level of HDL-C, and some of them decrease levels of LDL-C and TG in addition to increasing HDL- $\mathrm{C}^{[87]}$. However, the first clinical trial to investigate the effects of torcetrapib on atherosclerotic events was halted because of statistically higher incidences of death: hypertension due to activation of the renin-angiotensin-aldosterone system was considered to be a cause of the adverse events ${ }^{[87]}$. In the subsequent randomized clinical trials of CETP inhibitors including dalcetrapib ${ }^{[88]}$ and evacetrapib ${ }^{[89]}$, serious off-target adverse events did not occur, but no risk reduction for cardiovascular events was revealed. In a more recent randomized clinical trial ${ }^{[90]}, \geq 4$ years anacetrapib treatments significantly prevented the development of coronary artery events (HR, 0.91) associated with no increased risks of death, cancer, and other serious adverse events. It is necessary to examine whether CETP inhibitors are effective for preventing stroke.

Proprotein convertase subtilisin-kexin type 9 (PCSK9) is a hepatic protease, which decreases receptors for LDL in liver and causes an increase in the plasma concentration of LDL-C. A monoclonal antibody inhibitor of PCSK9 is a novel lipid-lowering agent, which has been reported to suppress LDL-C levels by $60 \%-70 \%$ in patients taking a statin ${ }^{[9-93]}$. A meta-analysis of 24 randomized clinical trials including 10,159 patients reported that a PCSK9 inhibitor significantly reduced all-cause mortality (OR, 0.45) and myocardial infarction $(\mathrm{OR}, 0.49)^{[91]}$. In a randomized clinical trial including 4,465 patients, evolocumab decreased LDL-C levels by $61 \%$ as well as the incidence of cardiovascular events (HR, 0.47) ${ }^{[92]}$. However, evolocumab treatment exhibited neurocognitive adverse events more frequently compared with the control group regardless of LDL-C levels during the treatment ${ }^{[92]}$. Another phase III randomized clinical trial including 2,341 patients with high risks for cardiovascular events revealed that alirocumab treatment induced a $62 \%$ reduction in a level of LDL-C, which in turn reduced a major adverse cardiovascular event compared with placebo treatment $(\mathrm{HR}, 0.52)^{[93]}$. However, alirocumab treatment did not decrease risks of ischemic strokes, and rather had higher rates of injection-site reactions, myalgia, and neurocognitive side effects including memory impairments, confusional states and ophthalmologic events ${ }^{[93]}$. The mechanisms of neurocognitive changes associated with PCSK9 inhibitors are not certain and the future clarification is required.

Randomized clinical trials have not been conducted and are needed to test the safety and efficacy of nonstatin lipid-lowering agents as add-on treatments to a statin in patients with CA stenosis.

\section{NON-DRUG THERAPIES}

In addition to medications, lifestyle changes are a requisite component of lipid-lowering therapies, including diet and exercise. According to the 2016 guidelines of the European Society of Cardiology and the European Atherosclerosis Society for the management of dyslipidemia, diet should include a reducing intake of saturated fats and increasing intake of polyunsaturated and monounsaturated fats, cereals, fruits, vegetables and fat free dairy products ${ }^{[94]}$. In addition, patients with a body mass index greater than $25 \mathrm{~kg} / \mathrm{m}^{2}$ should follow lowcalorie diets. Moreover, at least 30 min a day of moderate-intensity aerobic activity for five days a week are recommended. The intake of dietary fiber, monounsaturated and PUFAs has been reported to decrease stroke risks by reducing LDL-C and postprandial lipids levels with some discrepancies ${ }^{[95]}$. Habitual exercise has also been revealed to have beneficial effects on risk factors of stroke, including hypertension, dyslipidemia, diabetes and body weight: moderate or high exercise was associated with a significant reduction in the incidence of stroke or its mortality ${ }^{[96]}$. However, there is no evidence from published randomized controlled trials to support the secondary stroke prevention associated with post-stroke diet and exercise. 


\section{CONCLUSION}

Dyslipidemia is a major player in the formation and progression of CA atherosclerosis. Statins can improve serum lipid profiles and are beneficial for both of the primary and secondary prevention of ischemic stroke. In addition, statins exert beneficial effects on CA plaque stability as well as regression of CA IMT and plaque volume, causing reduction in the risk of perioperative complications related with CAS and CEA. Nonstatin lipid-lowering agents may have adjunctive effects as an add-on treatment to statin, and are expected further to suppress atherosclerotic CA diseases and to reduce stroke risks. However, the widespread effects of lipid-lowering agents on serum lipid profile and atherogenesis have not been fully elucidated. Further investigations to reveal the mechanisms of the effects and the randomized clinical trials to test the safety and efficacy of lipid-lowering agents are needed in patients with CA stenosis.

\section{DECLARATIONS}

\section{Authors' contributions}

Conception and design of the study, wrote the original draft: Miura Y

Provided administrative support, reviewed and edited the manuscript: Suzuki H

\section{Availability of data and materials}

Not applicable.

\section{Financial support and sponsorship}

None.

\section{Conflicts of interest}

All authors declared that there are no conflicts of interest.

\section{Ethical approval and consent to participate}

Not applicable.

\section{Consent for publication}

Not applicable.

\section{Copyright}

(c) The Author(s) 2019.

\section{REFERENCES}

1. Fine-Edelstein JS, Wolf PA, O’Leary DH, Poehlman H, Belanger AJ, et al. Precursors of extracranial carotid atherosclerosis in the Framingham study. Neurology 1994;44:1046-50.

2. Rockman CB, Hoang H, Guo Y, Maldonado TS, Jacobowitz GR, et al. The prevalence of carotid artery stenosis varies significantly by race. J Vasc Surg 2013;57:327-37.

3. The Intercollegiate Working Party for Stroke. National clinical guidelines for stroke. 2th ed. London: Royal College of Physicians; 2004.

4. Mark1 M, Wegent F, Zech T, Bauer S, Strecker C, et al. In vivo wall shear stress distribution in the carotid artery: effect of bifurcation geometry, internal carotid artery stenosis, and recanalization therapy. Circ Cardiovasc Imaging 2010;3:647-55.

5. Spence JD. Asymptomatic carotid stenosis. Circulation 2013;127:739-42.

6. Marquardt L, Geraghty OC, Mehta Z, Rothwell PM. Low risk of ipsilateral stroke in patients with asymptomatic carotid stenosis on best medical treatment: a prospective, population-based study. Stroke 2010;41:e11-7.

7. Raman G, Moorthy D, Hadar N, Dahabreh IJ, O’Donnell TF, et al. Management strategies for asymptomatic carotid stenosis: a systematic review and meta-analysis. Ann Intern Med 2013;158:676-85.

8. Blauw GJ, Lagaay AM, Smelt AH, Westendorp RG. Stroke, statins, and cholesterol. A meta-analysis of randomized, placebo-controlled, double-blind trials with HMG-CoA reductase inhibitors. Stroke 1997;28:946-50. 
9. Amarenco P, Bogousslavsky J, Callahan A 3rd, Goldstein LB, Hennerici M, et al. High-dose atorvastatin after stroke or transient ischemic attack. N Engl J Med 2006;355:549-59.

10. Hosomi N, Kitagawa K, Nagai Y, Nakagawa Y, Aoki S, et al. Desirable low-density lipoprotein cholesterol levels for preventing stroke recurrence: a post hoc analysis of the J-STARS study (Japan Statin Treatment Against Recurrent Stroke). Stroke 2018;49:865-71.

11. Prasad K. Do statins have a role in reduction/prevention of post- PCI restenosis? Cardiovasc Ther 2013;31:12-26.

12. Crouse JR, Byington RP, Bond MG, Espeland MA, Sprinkle JW, et al. Pravastatin, lipids, and atherosclerosis in the carotid arteries: design features of a clinical trial with carotid atherosclerosis outcome. Control Clin Trials 1992;13:495-506.

13. Espeland MA, O'Leary DH, Terry JG, Morgan T, Evans G, et al. Carotid intimal-media thickness as a surrogate for cardiovascular disease events in trials of HMG-CoA reductase inhibitors. Curr Control Trials Cardiovasc Med 2005;6:3.

14. Bedi US, Singh M, Singh PP, Bhuriya R, Bahekar A, et al. Effects of statins on progression of carotid atherosclerosis as measured by carotid intimal--medial thickness: a meta-analysis of randomized controlled trials. J Cardiovasc Pharmacol Ther 2010;15:268-73.

15. Koga M, Toyoda K, Minematsu K, Yasaka M, Nagai Y, et al. Long-term effect of pravastatin on carotid intima-media complex thickness: the J-STARS echo study (Japan Statin Treatment Against Recurrent Stroke). Stroke 2018;49:107-13.

16. Corti R, Fuster V, Fayad ZA, Worthley SG, Helft G, et al. Lipid lowering by simvastatin induces regression of human atherosclerotic lesions: two years' follow-up by high-resolution noninvasive magnetic resonance imaging. Circulation 2002;106:2884-7.

17. Ainsworth CD, Blake CC, Tamayo A, Beletsky V, Fenster A, et al. 3D ultrasound measurement of change in carotid plaque volume: a tool for rapid evaluation of new therapies. Stroke 2005;36:1904-9.

18. Tang TY, Howarth SP, Miller SR, Graves MJ, Patterson AJ, et al. The ATHEROMA (Atorvastatin Therapy: Effects on Reduction of Macrophage Activity) study. Evaluation using ultrasmall superparamagnetic iron oxide-enhanced magnetic resonance imaging in carotid disease. J Am Coll Cardiol 2009;53:2039-50.

19. Mujaj B, Bos D, Selwaness M, Leening MJG, Kavousi M, et al. Statin use is associated with carotid plaque composition: the Rotterdam study. Int J Cardiol 2018;260:213-8.

20. Prasad K. Pathophysiology of atherosclerosis. In: Chang JB, Olsen ER, Prasad K, Sumpio BE, editors. Textbook of Angiology. New Yor: Springer Verlag; 2000. pp. 85-105.

21. Prasad K. Pathophysiology and medical treatment of carotid artery stenosis. Int J Angiol 2015;24:158-72.

22. Orekhov AN, Ivanova EA. Introduction of the special issue "Atherosclerosis and Related Diseases". Vessel Plus 2017;1:163-5.

23. Schaffner T, Taylor K, Bartucci EJ, Fischer-Dzoga K, Beeson JH, et al. Arterial foam cells with distinctive immunomorphologic and histochemical features of macrophages. Am J Pathol 1980;100:57-80.

24. Hundal RS, Salh BS, Schrader JW, Gómez-Muñoz A, Duronio V, et al. Oxidized low density lipoprotein inhibits macrophage apoptosis through activation of the PI 3-kinase/PKB pathway. J Lipid Res 2001;42;1483-91.

25. Galis ZS, Sukhova GK, Lark MW, Libby P. Increased expression of matrix metalloproteinases and matrix degrading activity in vulnerable regions of human atherosclerotic plaques. J Clin Invest 1994;94:2493-503.

26. Moreno PR, Purushothaman KR, Zias E, Sanz J, Fuster V. Neovascularization in human atherosclerosis. Curr Mol Med 2006;6:457-77.

27. Carr S, Farb A, Pearce WH, Virmani R, Yao JS. Atherosclerotic plaque rupture in symptomatic carotid artery stenosis. J Vasc Surg 1996;23:755-65.

28. Morgan AR, Rerkasem K, Gallagher PJ, Zhang B, Morris GE, et al. Differences in matrix metalloproteinase-1 and matrix metalloproteinase-12 transcript levels among carotid atherosclerotic plaques with different histopathological characteristics. Stroke 2004;35:1310-5.

29. Montecucco F, Di Marzo V, da Silva RF, Vuilleumier N, Capettini L, et al. The activation of the cannabinoid receptor type 2 reduces neutrophilic protease-mediated vulnerability in atherosclerotic plaques. Eur Heart J 2012;33:846-56.

30. Rao VH, Rai V, Stoupa S, Subramanian S, Agrawal DK. Tumor necrosis factor- $\alpha$ regulates triggering receptor expressed on myeloid cells-1-dependent matrix metalloproteinases in the carotid plaques of symptomatic patients with carotid stenosis. Atherosclerosis 2016;248:160-9.

31. Fang Y, Mohler ER 3rd, Hsieh E, Osman H, Hashemi SM, et al. Hypercholesterolemia suppresses inwardly rectifying K+ channels in aortic endothelium in vitro and in vivo. Circ Res 2006;98:1064-71.

32. Morikage N, Kishi H, Sato M, Guo F, Shirao S, et al. Cholesterol primes vascular smooth muscle to induce Ca2 sensitization mediated by a sphingosylphosphorylcholine-Rho-kinase pathway: possible role for membrane raft. Circ Res 2006;99:299-306.

33. Amiya E. Interaction of hyperlipidemia and reactive oxygen species: insights from the lipid-raft platform. World J Cardiol 2016;8:689-94.

34. Podrez EA, Poliakov E, Shen Z, Zhang R, Deng Y, et al. Identification of a novel family of oxidized phospholipids that serve as ligands for the macrophage scavenger receptor CD36. J Biol Chem 2002;277:38503-16.

35. Zhang Q, Malik P, Pandey D, Gupta S, Jagnandan D, et al. Paradoxical activation of endothelial nitric oxide synthase by NADPH oxidase. Arterioscler Thromb Vasc Biol 2008;28:1627-33.

36. Borén J, Taskinen MR, Olofsson SO, Levin M. Ectopic lipid storage and insulin resistance: a harmful relationship. J Intern Med 2013;274:25-40.

37. Wang L, Gill R, Pedersen TL, Higgins LJ, Newman JW, et al. Triglyceride-rich lipoprotein lipolysis releases neutral and oxidized FFAs that induce endothelial cell inflammation. J Lipid Res 2009;50:204-13.

38. Welty FK. How do elevated triglycerides and low HDL-cholesterol affect inflammation and atherothrombosis? Curr Cardiol Rep 2013;15:400.

39. Botham KM, Moore EH, De Pascale C, Bejta F. The induction of macrophage foam cell formation by chylomicron remnants. Biochem 
Soc Trans 2007;35:454-8.

40. Chapman MJ, Ginsberg HN, Amarenco P, Andreotti F, Borén J, et al. Triglyceride-rich lipoproteins and high-density lipoprotein cholesterol in patients at high risk of cardiovascular disease: evidence and guidance for management. Eur Heart J 2011;32:1345-61.

41. Harangi M, Szentpéteri A, Nádró B, Lőrincz H, Seres I, et al. HDL subfraction distribution and HDL function in untreated dyslipidemic patients. Vessel Plus 2017;1:166-73.

42. Assmann G, Gotto AM Jr. HDL cholesterol and protective factors in atherosclerosis. Circulation 2004;109:III8-14.

43. Peshavariya H, Dusting GJ, Di Bartolo B, Rye KA, Barter PJ, et al. Reconstituted high-density lipoprotein suppresses leukocyte NADPH oxidase activation by disrupting lipid rafts. Free Radic Res 2009;43:772-82.

44. Calabresi L, Franceschini G, Sirtori CR, De Palma A, Saresella M, et al. Inhibition of VCAM-1 expression in endothelial cells by reconstituted high density lipoproteins. Biochem Biophys Res Commun 1997;238:61-5.

45. Mackness B, Hine D, Liu Y, Mastorikou M, Mackness M. Paraoxonase-1 inhibits oxidised LDL-induced MCP-1 production by endothelial cells. Biochem Biophys Res Commun 2004;318:680-3.

46. Navab M, Imes SS, Hama SY, Hough GP, Ross LA, et al. Monocyte transmigration induced by modification of low density lipoprotein in cocultures of human aortic wall cells is due to induction of monocyte chemotactic protein 1 synthesis and is abolished by high density lipoprotein. J Clin Invest 1991;88:2039-46.

47. Epand RM, Stafford A, Leon B, Lock PE, Tytler EM, et al. HDL and apolipoprotein A-I protect erythrocytes against the generation of procoagulant activity. Arterioscler Thromb 1994;14:1775-83.

48. Nofer JR, Walter M, Kehrel B, Wierwille S, Tepel M, et al. HDL3-mediated inhibition of thrombin-induced platelet aggregation and fibrinogen binding occurs via decreased production of phosphoinositide-derived second messengers 1,2-diacylglycerol and inositol 1,4,5-tris-phosphate. Arterioscler Thromb Vasc Biol 1998;18:861-9.

49. Imamura T, Doi Y, Arima H, Yonemoto K, Hata J, et al. LDL cholesterol and the development of stroke subtypes and coronary heart disease in a general Japanese population: the Hisayama study. Stroke 2009;40:382-8.

50. Kim YS, Sung HJ, Son SJ, Lim J, Kang YW, et al. Triglyceride (TG) down-regulates expression of MCP-1 and CCR2 in PMA-derived THP-1 macrophages. Genes Genomics 2013;35:125-30.

51. Labreuche J, Deplanque D, Touboul PJ, Bruckert E, Amarenco P. Association between change in plasma triglyceride levels and risk of stroke and carotid atherosclerosis: systematic review and meta-regression analysis. Atherosclerosis 2010;212:9-15.

52. Amarenco P, Labreuche J, Touboul PJ. High-density lipoprotein-cholesterol and risk of stroke and carotid atherosclerosis: a systematic review. Atherosclerosis 2008; 196:489-96.

53. Kontush A, Chapman MJ. Antiatherogenic function of HDL particle subpopulations: focus on antioxidative activities. Curr Opin Lipidol 2010;21:312-8.

54. Tiozzo E, Gardener H, Hudson BI, Dong C, Della-Morte D, et al. High-density lipoprotein subfractions and carotid plaque: the Northern Manhattan study. Atherosclerosis 2014;237:163-8.

55. Chei CL, Yamagishi K, Kitamura A, Kiyama M, Imano H, et al. High-density lipoprotein subclasses and risk of stroke and its subtypes in Japanese population: the circulatory risk in communities study. Stroke 2013;44:327-33.

56. Wang X, Dong Y, Qi X, Huang C, Hou L. Cholesterol levels and risk of hemorrhagic stroke: a systematic review and meta-analysis. Stroke 2013;44:1833-9.

57. Xie L, Wu W, Chen J, Tu J, Zhou J, et al. Cholesterol levels and hemorrhagic stroke risk in East Asian versus Non-East Asian populations: a systematic review and meta-analysis. Neurologist 2017;22:107-15.

58. Mathiesen EB, Joakimsen O, Bønaa KH. Prevalence of and risk factors associated with carotid artery stenosis: the Tromsø study. Cerebrovasc Dis 2001;12:44-51.

59. Raitakari OT, Juonala M, Kähönen M, Taittonen L, Laitinen T, et al. Cardiovascular risk factors in childhood and carotid artery intimamedia thickness in adulthood: the cardiovascular risk in young Finns study. JAMA 2003;290:2277-83.

60. Vouillarmet J, Helfre M, Maucort-Boulch D, Riche B, Thivolet C, et al. Carotid atherosclerosis progression and cerebrovascular events in patients with diabetes. J Diabetes Complications 2016;30:638-43.

61. Howard BV, Roman MJ, Devereux RB, Fleg JL, Galloway JM, et al. Effect of lower targets for blood pressure and LDL cholesterol on atherosclerosis in diabetes: the SANDS randomized trial. JAMA 2008;299:1678-89.

62. Kitagami M, Yasuda R, Toma N, Shiba M, Nampei M, et al. Impact of hypertriglyceridemia on carotid stenosis progression under normal low-density lipoprotein cholesterol levels. J Stroke Cerebrovasc Dis 2017;26:1793-800.

63. Laufs U, Gertz K, Dirnagl U, Böhm M, Nickenig G, et al. Rosuvastatin, a new HMG-CoA reductase inhibitor, upregulates endothelial nitric oxide synthase and protects from ischemic stroke in mice. Brain Res 2002;942:23-30.

64. Cheng ZJ, Dai TM, Shen YY, He JL, Li J, et al. Atorvastatin pretreatment attenuates ischemic brain edema by suppressing aquaporin 4. J Stroke Cerebrovasc Dis 2018;27:3247-55.

65. Zhu MX, Lu C, Xia CM, Qiao ZW, Zhu DN. Simvastatin pretreatment protects cerebrum from neuronal injury by decreasing the expressions of phosphor-CaMK II and AQP4 in ischemic stroke rats. J Mol Neurosci 2014;54:591-601.

66. Brott TG, Halperin JL, Abbara S, Bacharach JM, Barr JD, et al. 2011 ASA/ACCF/AHA/AANN/AANS/ACR/ASNR/CNS/SAIP/ SCAI/SIR/SNIS/SVM/SVS guideline on the management of patients with extracranial carotid and vertebral artery disease. A report of the American College of Cardiology Foundation/American Heart Association Task Force on Practice Guidelines, and the American Stroke Association, American Association of Neuroscience Nurses, American Association of Neurological Surgeons, American College of Radiology, American Society of Neuroradiology, Congress of Neurological Surgeons, Society of Atherosclerosis 
Imaging and Prevention, Society for Cardiovascular Angiography and Interventions, Society of Interventional Radiology, Society of NeuroInterventional Surgery, Society for Vascular Medicine, and Society for Vascular Surgery. Circulation 2011;124:e54-130.

67. Smilde TJ, van Wissen S, Wollersheim H, Trip MD, Kastelein JJ, et al. Effect of aggressive versus conventional lipid lowering on atherosclerosis progression in familial hypercholesterolaemia (ASAP): a prospective, randomised, doubleblind trial. Lancet 2001;357:577-81.

68. Corti R, Fuster V, Fayad ZA, Worthley SG, Helft G, et al. Effects of aggressive versus conventional lipid-lowering by simvastatin on human atherosclerotic lesions: a prospective, randomized, double-blind trial with high-resolution magnetic resonance imaging. J Am Coll Cardiol 2005;46:106-12.

69. Underhill HR, Yuan C, Zhao XQ, Kraiss LW, Parker DL, et al. Effect of rosuvastatin therapy on carotid plaque morphology and composition in moderately hypercholesterolemic patients: a high-resolution magnetic resonance imaging trial. Am Heart J 2008; $155: 584$

70. Lee JM, Wiesmann F, Shirodaria C, Leeson P, Petersen SE, et al. Early changes in arterial structure and function following statin initiation: quantification by magnetic resonance imaging. Atherosclerosis 2008;197:951-8.

71. Lenglet S, Quercioli A, Fabre M, Galan K, Pelli G, et al. Statin treatment is associated with reduction in serum levels of receptor activator of NF-kB ligand and neutrophil activation in patients with severe carotid stenosis. Mediators Inflamm 2014;2014:720987.

72. McGirt MJ, Perler BA, Brooke BS, Woodworth GF, Coon A, et al. 3-hydroxy-3-methylglutaryl coenzyme A reductase inhibitors reduce the risk of perioperative stroke and mortality after carotid endarterectomy. J Vasc Surg 2005;42:829-36.

73. Tanemura H, Maeda M, Ichikawa N, Miura Y, Umeda Y, et al. High-risk plaque for carotid artery stenting evaluated with 3-dimensional T1-weighted gradient echo sequence. Stroke 2013;44:105-10.

74. Texakalidis P, Giannopoulos S, Jonnalagadda AK, Chitale RV, Jabbour P, et al. Preoperative use of statins in carotid artery stenting: a systematic review and meta-analysis. J Endovasc Ther 2018;25:624-31.

75. De Caterina R, Scarano M, Marfisi R, Lucisano G, Palma F, et al. Cholesterol-lowering interventions and stroke: insights from a metaanalysis of randomized controlled trials. J Am Coll of Cardiol 2010;55:198-211.

76. Sorrentino SA, Besler C, Rohrer L, Meyer M, Heinrich K, et al. Endothelial-vasoprotective effects of high-density lipoprotein are impaired in patients with type 2 diabetes mellitus but are improved after extended-release niacin therapy. Circulation 2010;121:110-22.

77. Villines TC, Stanek EJ, Devine PJ, Turco M, Miller M, et al. The ARBITER 6-HALTS trial (arterial biology for the investigation of the treatment effects of reducing cholesterol 6-HDL and LDL treatment strategies in atherosclerosis): final results and the impact of medication adherence, dose, and treatment duration. J Am Coll Cardiol 2010;55:2721-6.

78. Lavigne PM, Karas RH. The current state of niacin in cardiovascular disease prevention: a systematic review and meta-regression. J Am Coll Cardiol 2013;61:440-6.

79. Bloomfield Rubins H, Davenport J, Babikian V, Brass LM, Collins D, et al. Reduction in stroke with gemfibrozil in men with coronary heart disease and low HDL cholesterol: the veterans affairs HDL intervention trial (VA-HIT). Circulation 2001;103:2828-33.

80. Jun M, Foote C, Lv J, Neal B, Patel A, et al. Effects of fibrates on cardiovascular outcomes: a systematic review and meta-analysis. Lancet 2010;375:1875-84.

81. Ishibashi S, Arai H, Yokote K, Araki E, Suganami H, et al. Efficacy and safety of pemafibrate (K-877), a selective peroxisome proliferator-activated receptor $\alpha$ modulator, in patients with dyslipidemia: results from a 24-week, randomized, double blind, activecontrolled, phase 3 trial. J Clin Lipidol 2018;12:173-84.

82. Calvo MJ, Martínez MS, Torres W, Chávez-Castillo M, Luzardo E, et al. Omega-3 polyunsaturated fatty acids and cardiovascular health: a molecular view into structure and function. Vessel Plus 2017:1:116-28.

83. Thies F, Garry JM, Yaqoob P, Rerkasem K, Williams J, et al. Association of n-3 polyunsaturated fatty acids with stability of atherosclerotic plaques: a randomised controlled trial. Lancet 2003;361:477-85.

84. Cawood AL, Ding R, Napper FL, Young RH, Williams JA, et al. Eicosapentaenoic acid (EPA) from highly concentrated n-3 fatty acid ethyl esters is incorporated into advanced atherosclerotic plaques and higher plaque EPA is associated with decreased plaque inflammation and increased stability. Atherosclerosis 2010;212:252-9.

85. Cannon CP, Blazing MA, Giugliano RP, McCagg A, White JA, et al. Ezetimibe added to statin therapy after acute coronary syndromes. N Engl J Med 2015;372:2387-97.

86. Bohula EA, Wiviott SD, Giugliano RP, Blazing MA, Park JG, et al. Prevention of stroke with the addition of ezetimibe to statin therapy in patients with acute coronary syndrome in IMPROVE-IT (improved reduction of outcomes: vytorin efficacy international trial). Circulation 2017;136:2440-50.

87. Barter PJ, Caulfield M, Eriksson M, Grundy SM, Kastelein JJ, et al. Effects of torcetrapib in patients at high risk for coronary events. N Engl J Med 2007;357:2109-22.

88. Schwartz GG, Olsson AG, Abt M, Ballantyne CM, Barter PJ, et al. Effects of dalcetrapib in patients with a recent acute coronary syndrome. N Engl J Med 2012;367:2089-99.

89. Lincoff AM, Nicholls SJ, Riesmeyer JS, Barter PJ, Brewer HB, et al. Evacetrapib and cardiovascular outcomes in high-risk vascular disease. N Engl J Med 2017;376:1933-42.

90. HPS3/TIMI55-REVEAL Collaborative Group, Bowman L, Hopewell JC, Chen F, Wallendszus K, et al. Effects of anacetrapib in patients with atherosclerotic vascular disease. N Engl J Med 2017;377:1217-27.

91. Dadu RT, Ballantyne CM. Lipid lowering with PCSK9 inhibitors. Nat Rev Cardiol 2014;11:563-75.

92. Navarese EP, Kolodziejczak M, Schulze V, Gurbel PA, Tantry U, et al. Effects of proprotein convertase subtilisin/kexin type 9 antibodies 
in adults with hypercholesterolemia: a systematic review and meta-analysis. Ann Intern Med 2015;163:40-51.

93. Robinson JG, Farnier M, Krempf M, Bergeron J, Luc G, et al. Efficacy and safety of alirocumab in reducing lipids and cardiovascular events. N Engl J Med 2015;372:1489-99.

94. Catapano AL, Graham I, De Backer G, Wiklund O, Chapman MJ, et al. 2016 ESC/EAS guidelines for the management of dyslipidaemias: the task force for the management of dyslipidaemias of the European Society of Cardiology (ESC) and European Atherosclerosis Society (EAS) developed with the special contribution of the European Association for Cardiovascular Prevention \& Rehabilitation (EACPR). Atherosclerosis 2016;253:281-344.

95. Iacoviello L, Bonaccio M, Cairella G, Catani MV, Costanzo S, et al. Diet and primary prevention of stroke: systematic review and dietary recommendations by the ad hoc Working Group of the Italian Society of Human Nutrition. Nutr Metab Cardiovasc Dis 2018;28:309-34.

96. Lee IM, Hennekens CH, Berger K, Buring JE, Manson JE. Exercise and risk of stroke in male physicians. Stroke 1999;30:1-6. 